\title{
L'impératif en français et en anglais contemporains
}

\begin{abstract}
As a deontic mode of enunciation, the imperative excludes any operation which implies a certain degree of agreement between speaker and addressee - presupposition, referential construction, etc. In order to make up for this fundamental disjunction, the speaker resorts to a range of compensatory devices such as the use of person desinences, the ethical dative, the position of clitic pronouns or the function of the first person plural. Yet an imperative utterance is also related to the declarative context in which it occurs, and this sometimes causes co-locution to give way to co-enunciation - witness the problem of negative imperatives, or markers of discourse consistency such as voyons in French. This twofold theoretical approach is here presented as applying to the French language, and then put to the test of the English language.
\end{abstract}

\section{Les trois modalités d'énonciation}

Dans une situation de communication directe, un locuteur s'adresse à un co-locuteur, qui, lorsqu'il prend la parole, devient à son tour locuteur. Le terme de co-locution sera donc utilisé ici chaque fois qu'il s'agira de désigner la situation de co-présence des interlocuteurs.

Les énoncés («utterances») pris en considération dans cet article sont analysables comme un dictum (relation prédicative) affecté d'un modus. Selon qu'ils sont de forme déclarative, interrogative ou impérative ${ }^{1}$, la nature du modus et la valeur de son support varient. Nous dirons que le modus est ontique si celui qui parle pose l'existence d'un événement ou exprime une

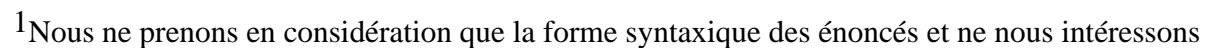
pas ici à la valeur illocutoire de certaines formes syntaxiques (par exemple énoncé interrogatif pour exprimer une injonction du type Voudrais-tu me passer le sel? Tu peux m'aider? etc.).
} 
opinion, déontique s'il énonce sa volonté de voir se produire une modification dans la situation co-locutoire.

Quant au support de ce modus, nous le désignerons par le terme d'énonciateur si le modus représente le point de vue de celui qui parle, et par celui de co-énonciateur s'il s'agit de la pensée que l'énonciateur prête à celui auquel il s'adresse. Le terme de co-énonciation sera donc utilisé chaque fois qu'il sera question d'identifier le support du modus.

1.1. La modalité qui affecte un énoncé de forme déclarative est ontique. L'énonciateur situe l'opinion qu'il énonce ou le phénomène dont il pose l'existence par rapport à la pensée qu'il prête au co-énonciateur. Il définit un état de la co-énonciation qui oscille entre trois valeurs : consensualité, discordance ${ }^{2}$, «objectivité». Ceci se marque notamment dans la rhétorique des présentatifs pour lesquels les pronoms définissent la valeur de la relation énonciateur/co-énonciateur plutôt que des références actancielles. Entre Vous avez le RER qui passe à Châtelet (prise à témoin consensuelle) et Il y a le RER qui passe à Châtelet (objectivité), c'est la relation énonciateur/coénonciateur qui s'est infléchie. Ici, énonciateur et co-énonciateur sont des pôles non séparables définissant le support de la modalité ontique.

1.2. L'énoncé de forme interrogative est affecté d'une modalité ontique «suspendue». En général, dans les questions totales, cette suspension se marque au niveau de la modalité d'ensemble par l'inversion sujet-verbe ou par l'intonation finale montante. Dans les questions partielles, elle se joue au niveau de la détermination des arguments du dictum : le pronom ou le syntagme interrogatif constitue un argument dont la référence est tout ensemble garantie mais incomplètement définie (Qui vois-tu? / Quel jour sommes-nous demain?). C'est de cette incomplétude que naît l'effet de suspension.

\footnotetext{
${ }^{2}$ Pour traduire une modification dans la consensualité co-énonciative préalablement établie, il nous semble plus juste de parler de «discordance» de points de vue, plutôt que de «rupture», terme que nous avons utilisé dans nos études antérieures (cf. L. Danon-Boileau «Ce que ça veut dire ...» et M.-A. Morel «Les présentatifs en français», in La deixis. Colloque en Sorbonne, Paris, PUF, 1992. pp. 415-426 et 507-518) et qui nous semble aujourd'hui trop restrictif.
} 
L'inachèvement ainsi construit par l'interrogation est adressé à celui qui écoute. S'inscrit alors en filigrane le support de la réponse à venir, lequel, pour les questions «vraies», jouit d'une certaine indépendance. Nous dirons que c'est au co-locuteur qu'il revient d'achever l'énoncé de forme interrogative.

L'interrogatif présente donc un statut mixte, relevant à la fois de la coénonciation et de la co-locution.

1.3. L'énoncé de forme impérative, enfin, est associé à une modalité déontique. Il s'inscrit directement dans la co-locution. Il n'est nullement fondé sur la co-énonciation. Nous ferons l'hypothèse que la relation locuteur/co-locuteur interdit certaines opérations fondées sur la coénonciation, et notamment diverses constructions ontiques et référentielles.

1.4. Les énoncés de forme impérative, déclarative et interrogative s'opposent de plusieurs manières 3 .

Du point de vue des conditions de production du discours, l'interrogatif et l'impératif s'opposent conjointement au déclaratif. L'impératif comme l'interrogatif exigent, en effet, une relation directe entre les co-locuteurs, ce qui n'est pas nécessairement le cas du déclaratif.

Du point de vue de la nature de la modalité, l'interrogatif et le déclaratif, tous deux de l'ordre de l'ontique, s'opposent à l'impératif, lequel est déontique.

\footnotetext{
${ }^{3}$ Soulignons ici notre dette envers J. Damourette et E. Pichon, qui tout au long de leur ouvrage, différencient très clairement trois plans énonciatifs: 1) le plan locutoire - où ils situent l'impératif, l'interjection, le vocatif et l'exclamation nominale - qui met en prise directe les deux pôles de l'échange (locuteur et allocutaire «sont tellement impliqués dans le fait langage qu'ils n'ont pas besoin d'être exprimés par des termes spéciaux»); 2) le plan allocutoire - où le locuteur, par le recours à l'interrogation, laisse à l'allocutaire la faculté de compléter le jugement laissé en suspens, 3) le plan délocutoire - où le locuteur parle du monde et où «les personnes ne figurent plus que comme des choses dont on raconte l'histoire et au sujet desquelles on exprime des jugements.». Ils opèrent ainsi une distinction très nette entre les participants directs de l'échange (locuteur et allocutaire) et leur représentation linguistique au plan délocutoire (le locutif $j e$ et l'allocutif $t u$ n'étant pour eux que des cas particuliers du délocutif $i l$ ). (Damourette et Pichon IV, § 1383, 1431 et 1432).
} 
Du point de vue de l'achèvement des opérations, l'impératif et le déclaratif s'opposent conjointement à l'interrogatif, puisqu'il est seul à demeurer «suspendu», ce que confirment les indices intonatifs, par exemple.

Du point de vue de la nature du support du modus, enfin, chacune des trois modalités se distingue des autres.

Le support du modus du déclaratif se calcule par rapport à la coénonciation.

Le support du modus de l'interrogatif se calcule également par rapport à la co-énonciation. Mais, du fait de l'appel à la réponse, il y a mise en jeu implicite d'un co-locuteur.

Le support du modus de l'impératif est réduit au seul locuteur, hors de toute co-énonciation. L'énoncé est simplement adressé à un co- locuteur.

\section{A. L'impératif en français}

On peut regrouper l'ensemble des questions que soulève l'énoncé de forme impérative en français sous trois chefs principaux.

\section{Questions suscitées par l'impératif}

2.1. Tout d'abord, quelle valeur attribuer à la marque de personne associée au verbe? Dans un énoncé de forme impérative, l'expression d'un sujet nominal ou pronominal est impossible, cependant que le verbe est affecté d'une marque de personne, laquelle en français est en outre variable (cf. l'alternance : sors / sortons / sortez). A ce problème d'ensemble on pourra associer la question du datif éthique (Ferme-moi cette porte), qui, à l'impératif, est réservé à la première personne, contrairement à ce qui se passe dans un énoncé de forme déclarative (Il lui a appris sa leçon en un rien de temps).

2.2. Se pose également la question de la position des pronoms clitiques. A l'impératif positif, ils se placent après le verbe (Donne-le moi) et non avant comme au déclaratif (*Me le donne). En revanche cet ordre peut (cf. § 3.2) être modifié quand l'impératif est associé à la négation (Ne me le donne pas). 
2.3. On peut enfin se pencher sur la question de l'articulation entre l'impératif et la trame déclarative. En règle générale, l'énoncé de forme impérative constitue un isolat dans la trame discursive; mais cette isolation n'est pas pure parataxe, et il convient de saisir comment la co- locution en arrive à s'infléchir partiellement en co-énonciation. On analysera pour ce faire la valeur de marqueurs tels que donc et voire. On montrera aussi comment certains impératifs (allons, écoute, disons) sont en fait devenus des marques de cohérence discursive et de redéfinition de la co-énonciation.

\section{Propriétés de l'énoncé impératif}

Notre analyse d'ensemble partira de l'idée que l'énoncé de forme impérative, compte tenu de la situation de co-locution qui le caractérise, se voit interdire diverses opérations - constructions référentielles notamment. En effet, pour construire une référence - celle d'un pronom sujet par exemple - il faut pouvoir postuler une situation de partage co- énonciatif avec celui auquel on s'adresse.

3.1. L'absence de détermination dans le vocatif ( $\varnothing$ Garçon!), l'emploi jussif d'un constituant nominal sans détermination ( $\varnothing$ Pinces!) ou le verbe sans sujet (Ø Donne!) sont autant de faits qui soulignent que la relation coénonciative n'est pas suffisamment établie pour servir de fondement aux constructions référentielles. Il convient toutefois de noter que, dans la colocution, il faut que le locuteur s'assure que le co-locuteur s'est bien identifié comme tel. Ainsi s'explique, à notre avis, qu'on ait parfois recours à une fiction de co-énonciation pour construire une référence à laquelle le colocuteur peut s'identifier, ce qui se traduit en français par la présence d'un déterminant devant le vocatif nominal (Eh! le grand au fond de la salle, viens effacer le tableau!), et en anglais par l'expression d'un sujet devant l'impératif (cf. $\S 4$ et 5).

Reste que dans l'énoncé de forme impérative, le verbe demeure affecté d'une marque de personne dont il faut préciser l'effet. On proposera de la lire comme un indice défini unilatéralement par le locuteur afin de signaler quelle serait la valeur de l'argument sujet susceptible d'instancier la relation prédicative, bien que sa référence, faute de partage co-énonciatif, ne puisse 
être totalement construite. La modalité déontique qui affecte l'ensemble de l'énoncé n'autorise pas la construction référentielle du sujet ${ }^{4}$.

3.1.1. L'absence de sujet à l'impératif permet de contraster dans le registre déontique les énoncés de type «Toi, mange ta soupe!» avec ceux de type «Tu manges ta soupe!»5. Les premiers marquent une co-locution sans coénonciation - le vocatif construit un co-locuteur à partir de la situation colocutoire -, tandis que l'énoncé de forme déclarative à la deuxième personne constitue un forçage de consensualité co-énonciative. La présence du sujet $t u$ avant le verbe assigne explicitement au co- énonciateur le statut d'argument dans une relation prédicative ontiquement validée. Ceci explique pourquoi un énoncé de ce type est généralement interprété comme plus contraignant, voire plus brutal, que l'énoncé de forme impérative. Au fond, un ordre à la forme impérative n'engage que son auteur. En revanche, fût-ce à son corps défendant, un énoncé de forme déclarative engage le co-énonciateur.

3.1.2. Il convient également d'apprécier la différence de valeur entre sujet détaché dans les énoncés de forme déclarative d'une part, et vocatif associé aux énoncés de forme impérative de l'autre.

$\mathrm{Au}$ plan déclaratif, les opérations référentielles sont supportées conjointement par l'énonciateur et le co-énonciateur. Un pronom de deuxième personne placé en position détachée acquiert de ce fait nécessairement une valeur de thème et ne peut jamais être interprété comme un vocatif (cf. vous dans l'exemple suivant). Cette mise en position thématique du pronom ne crée pas de rupture dans la continuité coénonciative elle-même. Lorsque l'énonciateur souhaite interrompre le fil du

\footnotetext{
${ }^{4}$ Ici encore nous rejoignons les vues proposées par Damourette et Pichon, pour qui la marque de seconde personne de l'impératif évoque l'allocutaire «non pas comme l'objet d'une affirmation judicative» - c'est-à-dire non pas comme un argument pourvu d'une référence fondée sur un jugement partagé avec l'autre- mais comme «la matière d'un façonnement volontaire» - c'est-à-dire comme l'effet d'un décret émanant du seul locuteur. (Damourette et Pichon IV, § 1432).

${ }^{5}$ Nous ne sommes pas en mesure ici d'aborder la question de la présence en ancien français du pronom sujet de 2e personne, avant ou après l'impératif, qui est diversement interprétée selon les grammairiens et les linguistes (cf. en particulier F. Brunot \& C. Bruneau, Kr. Nyrop et R.L. Wagner) et qui nécessiterait une étude à partir des textes.
} 
récit «déclaratif» par un vocatif, il doit être fait appel au nom propre (ou à une expression nominale non déterminée).

(1) alors je sais bien que vous Marguerite Duras vous avez connu d'autres

Il n'en va pas exactement de même avec l'impératif. Un pronom de deuxième personne, placé en position détachée dans un énoncé de forme impérative, est généralement interprété comme un vocatif, et non comme un thème (Toi, viens ici! Vous, taisez-vous!).

(2) Et vous, Iphicrate, appelez-vous Arlequin ou bien Hé (Marivaux)

3.1.3. Venons-en à présent aux conséquences positives du centrage sur le locuteur. On notera tout d'abord la présence fréquente d'un pronom datif (dit éthique) de première personne (Range-moi ta chambre. Va me faire tes devoirs tout de suite.), lequel ne se rencontre à l'impératif qu'à la première personne, alors que, dans un énoncé de forme déclarative, il est admissible aux autres personnes (Son fils lui a fait une rougeole cet hiver. Il te mange dix bananes de suite!).

3.1.4. A ces considérations nous souhaiterions ajouter quelques remarques sur la valeur de l'impératif première personne du pluriel. Tout d'abord, le statut spécifique des énoncés de forme impérative exclut la possibilité de mettre en jeu une valeur de -ons qui serait du type «je + il». En effet, ceci supposerait la construction préalable d'une troisième personne, laquelle, comme Damourette et Pichon (IV § 1431-1432) l'ont montré, repose sur une désignation conjointe supportée par la co- énonciation. A l'impératif première personne du pluriel, le sujet potentiel de la validation de la relation prédicative est associé au locuteur. La distance entre les co-locuteurs est de ce fait réduite et l'association du locuteur à l'ensemble des opérations énonciatives plus forte.

On ne s'étonnera donc pas de constater que la première personne du pluriel soit une manière de renforcer la déonticité de l'énoncé de forme impérative.

De ce point de vue, la différence entre les énoncés de forme impérative à la deuxième personne et à la première personne est très éclairante. Dégageons le couloir! (dit par un receveur d'autobus à une grosse dame, cf. succès (Interview de M. Duras par B. P 
Brunot et Bruneau 1969, 323) sera interprété comme une injonction plus forte que Dégagez le couloir!. 
Il est donc normal que la première personne soit utilisée dans les situations pédagogiques (Prenons le cas de .... Venons-en au fait.) et d'une manière générale dans toutes les situations où la déonticité est privilégiée (morale d'une fable, etc).

(3) La fortune se plaît à faire de ces coups:

Tout vainqueur insolent à sa perte travaille.

Défions-nous du sort, et prenons garde à nous

Après le gain d'une bataille. (La Fontaine, Les deux coqs)

Dans d'autres situations, le recours à la première personne permet de souligner que l'initiative de la validation de la relation prédicative envisagée revient au locuteur.

(4) Camille - ... viens, portons-lui secours; ...

Perdican - Non, en vérité, je n'entrerai pas.... Vas-y, Camille,

et tâche de la ramener. (Musset, On ne badine pas ...)

(5) Couvrons-nous, Grands d'Espagne! (Hugo, Hernani)

L'impératif première personne du pluriel peut même renvoyer au seul locuteur (Réfléchissons). L'effet de théâtralité qui apparaît souvent provient précisément du renforcement de la déonticité.

(6) Trivelin - Souvenez-vous en prenant son nom, mon cher ami, qu'on vous Arlequin - Oui, oui, corrigeons, corrigeons! Iphicrate - Maraud! (Marivaux, L'île des esclaves)

3.1.5. Nous ne saurions terminer cette étude des marques personnelles sans évoquer le cas des formes verbales à l'impératif qui, en français contemporain, se sont figées, sans pour autant perdre totalement le modus déontique ni l'autonomie propres à l'énoncé de forme impérative. Citons, entre autres, à la deuxième personne : «allez, attends, attendez, avoue, croismoi, dis, dis donc, dites-moi, écoute, écoutez, excuse-moi, note, notez, pensez donc, remarque, tiens, tenez, va, voyez», et à la première personne : «allons, disons, mettons, voyons, voyons voir(e)».

Certains de ces impératifs ont acquis le statut de véritables interjections. D'autres au contraire, placés soit à l'initiale d'un énoncé, soit au cours de son déroulement (en incise), ont un statut d'indice de cohérence discursive. Ils ont pour rôle de redéfinir l'état de la co-énonciation, et cela grâce à la marque de personne qui les affecte (cf. $\S 1.1$ le rôle des pronoms dans les présentatifs personnels). Avec la deuxième personne (va! allez! voyez!) l'énoncé se fait le donne bien moins pour réjouir votre 
plus persuasif, il témoigne de plus de sollicitude à l'égard de l'autre : il y a recherche d'une forme de consensualité, alors que l'emploi de la première personne (allons! voyons!) exprime plutôt la désapprobation du locuteur face à la situation : il y a soulignement d'une discordance.

(7) Vladimir - Voyons, Gogo, ne sois pas comme ça. (...)

Estragon - Fais-moi penser d'apporter une corde demain.

Vladimir - Oui. Viens (...) N'y pense plus, va. Viens.

(Becket, En attendant Godot)

Cela permet aussi de comprendre le rôle spécifique du marqueur de reformulation disons dans un énoncé déclaratif, par opposition à celui de je veux dire ou de enfin. Loin de marquer la consensualité dans la recherche d'une formulation adéquate, disons souligne plutôt une prise en charge égocentrée de l'énoncé qu'il introduit.

(8) mais c'que j'constate moi c'est que:_y a quelques années y a vingt ans disons dix ans vingt ans__ les gamins d'sixième qui en moyenne étaient plus jeunes franchissaient ça e: allaient plus facilement et plus vite (Conversation 1991)

3.2. Venons-en à présent à la seconde question, celle qui a trait à l'emplacement des pronoms. A l'impératif, les divers pronoms compléments se placent après le verbe, même si l'on observe un certain flottement dans leur ordre de succession (Donne-le moi / Donne-moi le / Donne-m'en / Donne-z-en moi / Donne-moi-z-en) ${ }^{6}$.

3.2.1. La comparaison de la place des pronoms personnels dans un énoncé de forme impérative, interrogative et déclarative invite à lire leur position par rapport au verbe comme un indice de leur statut référentiel et thématique : lorsque le pronom précède le verbe dans la chaîne, c'est qu'il dispose d'un statut thématique qui peut lui assurer une référence; s'il suit le verbe, c'est que son statut référentiel ou thématique n'est pas suffisamment établi; il s'ensuit alors une suspension de modalité ontique.

\footnotetext{
${ }^{6}$ Notons que seules la première et la troisième de ces formes sont retenues par les grammairiens normatifs, les autres étant considérées comme appartenant au langage «relâché». Nous intéressant ici aux séquences produites en situation de communication directe, nous avons jugé nécessaire de retenir toutes les réalisations possibles.
} 
A l'interrogation totale, cette suspension est provisoire, puisque le colocuteur est sollicité pour opérer la validation 7 .

A l'impératif la suspension est définitive puisque la relation verbe- objet est la cible d'une modalité déontique.

3.2.2. Cette façon d'analyser l'ordre verbe-objet à l'impératif serait évidemment incomplète si l'on ne prenait pas en compte la modification de l'ordre des mots qui apparaît à la négation (Lis-les / Ne les lis pas / *Ne lisles pas / Les lis pas / Lis-les pas).

A première vue, il peut paraître surprenant que ce soit dans le registre de la négation que l'on retrouve l'ordre des éléments du déclaratif. En réalité, toute négation implique un préalable de co-énonciation, de partage présuppositionnel. A l'impératif comme au déclaratif, la négation implique un contenu de pensée pris en charge conjointement par l'énonciateur et le coénonciateur, le différend ne se marquant que sur la valeur positive ou négative associée au prédicat. Le rôle du discordanciel ne est précisément de signaler l'existence d'un contenu de pensée consensuellement partagé, à partir de quoi l'énonciateur peut construire une discordance. Il est de fait impossible de postposer les pronoms en présence du discordantiel (*N'interrompez-moi pas).

(9) Ne m'interrompez point, mes enfants. (Marivaux)

La mise en jeu de la négation rapproche ainsi l'impératif du déclaratif. Sans doute est-ce l'infléchissement opéré par la négation qui permet de saisir que si un impératif passif est impossible (comment faire porter une injonction sur un état?), la combinaison avec la négation conduit à un énoncé acceptable (Ne sois pas bouleversé par cette nouvelle / *Sois bouleversé par cette nouvelle) 8 .

L'impératif négatif occupe ainsi une position variable, située à michemin entre le plan du déclaratif (de la co-énonciation), et le plan de

\footnotetext{
${ }^{7}$ En l'absence de l'inversion du sujet, la suspension de la modalité ontique dans les séquences interrogatives est toujours marquée (cf. la présence d'un mot interrogatif dont la référence est incomplètement définie, le point d'interrogation à l'écrit et l'intonation haute à l'oral).

${ }^{8}$ L'impératif passif est toutefois possible avec une valeur performative et quasi «magique»cf. Soyez-en remercié. Sois rassuré. Sois transformé en crapaud.
} 
l'impératif pur (de la co-locution). Et de fait, sitôt que le discordanciel est omis, et qu'il n'y a plus d'indication explicite d'un contenu de pensée consensuellement partagé, les pronoms peuvent ${ }^{9}$ reprendre la place qui est la leur à l'impératif affirmatif, l'élément forclusif de la négation pas se plaçant après le groupe «V+Pro» (Touche-le pas. Dis-lui pas.).

3.2.3. C'est sans doute en relation avec le statut référentiel des pronoms qu'il convient d'analyser la marque /z/ qui apparait comme liaison entre le verbe à l'impératif et les pronoms anaphoriques en et $y$ : «parle-z-en». D'ordinaire, cette marque est conçue comme extension analogique de la marque $-s$ de 2e personne (Prends-en. Conduis-y les enfants. Cf. entre autres, Nyrop 1960, 120). En réalité, cette liaison en /z/ se retrouve aux trois personnes de l'impératif (vas-y; allons-y; allez-y; parles-en; parlons-en; parlez-en), ce qui contrevient à cette façon de voir.

Pour notre part, nous l'analysons comme un effet de l'impératif en tant que tel, plutôt que comme un effet de la 2e personne. Il convient alors d'en préciser la raison d'être.

On a souligné tantôt que la place des pronoms derrière le verbe était liée à la suspension de la modalité ontique et que cette suspension avait une incidence directe sur la validation de la référence du pronom. On sera alors porté à interpréter la liaison en /z/ comme une façon de pallier le défaut de fondement des constructions référentielles pronominales, en raccrochant l'objet sémantique - en ou $y$ - à tout indice direct ou indirect du support du modus - le verbe ou le pronom lère personne, mais non celui de $3 \mathrm{e}$ personne (Donne-z-en moi / Donne-moi-z-en /*Donne- lui-z-en) 10 .

3.3. Dans ce qui précède, l'impératif a été rapporté à une relation de colocution dépourvue de toute consensualité co-énonciative. On a posé qu'il en résultait une sorte d'isolation de l'énoncé de forme impérative lui même ${ }^{11}$.

\footnotetext{
${ }^{9}$ Notons que l'ordre «Pro+V» se rencontre même en l'absence du discordantiel (Les lis pas).

${ }^{10}$ Pour le registre de langage de ces séquences, cf. remarque note 6.

${ }^{11}$ De fait, un énoncé de forme impérative se présente souvent comme discursivement autonome, et à l'oral, cette autonomie se marque d'une part par une courbe intonative descendante, et d'autre part par une syllabe finale de niveau bas. Lorsqu'il apparaît ainsi, l'énoncé de forme impérative ne peut guère être coordonné à un déclaratif ou à un interrogatif.
} 
On a vu toutefois que cette configuration s'infléchissait avec l'impératif négatif.

Il convient d'aller plus avant dans l'analyse de l'articulation entre déclaratif et impératif. Pour ce faire, nous examinerons la valeur de certains marqueurs qui leur sont communs, afin de cerner la façon dont ils permettent d'infléchir une relation de co-locution en relation de co- énonciation partielle.

3.3.1. Donc. Dans un énoncé de forme déclarative, l'énonciateur souligne avec donc qu'il opère un recentrage thématique, sans pour autant rompre la consensualité co-énonciative. Placé à l'initiale de l'énoncé, il a souvent un statut de «ligateur discursif conclusif»(cf. Donc Socrate est un homme.). Cette position et ce statut lui sont refusés à l'interrogatif et à l'impératif. En revanche, son insertion est possible derrière le marqueur interrogatif et/ou derrière le verbe (Qui donc? Où est-il donc parti? Parle donc!). Cette position confère à donc un statut particulier d'indice de recentrage dans le cadre de la co-locution, d'ajustement entre les co- locuteurs. Ceci explique que l'impératif suivi de donc soit interprété comme une invite plus pressante, comme un renforcement de l'injonction (Entrez donc! Mange donc au lieu de parler!).

3.3.2. Voire / Voir. L'adverbe voire présente normalement un $e$ final, lié à son étymologie (latin vero «vraiment, en vérité»). Toutefois dès les plus anciens textes français, il s'est produit une confusion - ou une fusion (cf. Damourette et Pichon VI, § 2177) - avec le verbe voir, explicable par l'homophonie de ces deux lexèmes, par leur position postverbale et par la proximité de leurs effets de sens.

Voir $(e)$ dans «Dis voir(e)» ou «Donne voir(e)» est en effet interprétable comme un adverbe ou comme un infinitif de but, dont le support est le locuteur («pour que je puisse voir»). Le soulignement égocentré opéré par voir (e) apparaît encore plus clairement lorsqu'on l'oppose à donc (Ecoute-moi voir(e) / Ecoute-moi donc ou Dis voir(e) / Dis donc). A la différence de donc, qui signale un ajustement avec le co-locuteur, voir(e) implique une discordance. Dans un énoncé tel que «Mange voir(e)», il est interprétable

Nous laissons de côté ici la question de l'impératif hypothétique (Libérez-le et c'est la révolution demain). 
comme une sorte de défi lancé au co-locuteur («si tu l'oses») ou en tout cas comme un renforcement de l'intérêt du locuteur («que je voie ce que cela donne»).

(10) Dis voire comment il s'appelle, le docteur? (Mme XW)

Regardez voire s'il est pointé déjà, avant de rouspéter

(Receveur d'omnibus, in D \& P, VI, § 2175)

\section{B. Le sujet de l'énoncé impératif en anglais}

Cette seconde partie a pour objectif de définir les points de recoupement et de divergence entre le français et l'anglais concernant les domaines évoqués en $\mathrm{A}^{12}$. Si l'analyse avancée sur le fonctionnement de l'impératif comme modalité discursive en français paraît transposable quasiment terme à terme en l'anglais, nous souhaitons ici étudier en détail l'apparente incompatibilité des hypothèses sur les marques de personne associées au verbe avec les faits de langue attestés e anglais : contrairement au français, en effet, l'anglais accepte la présence d'un sujet dans les énoncés de forme impérative.

Après avoir brièvement différencié sujet et vocatif et étudié divers phénomènes intonatifs, afin de pouvoir travailler sur des énoncés de forme impérative où la présence d'un sujet est indiscutable, nous passerons en revue les divers types de groupes nominaux pouvant occuper cette fonction pour voir si les faits de langue ainsi mis à jour peuvent être traités selon la grille de lecture proposée pour le français.

\section{Typologie}

Les groupes nominaux $2^{\mathrm{e}}$ ou $3^{\mathrm{e}}$ personne apparaissant dans un énoncé de forme impérative sont souvent ressentis comme faisant tous référence à une $2^{\mathrm{e}}$ personne, d'où l'opinion couramment répandue selon laquelle le sujet d'un énoncé de forme impérative est en fait un vocatif. Par ailleurs, s'ils sont bien perçus comme des sujets $3^{\mathrm{e}}$ personne, l'énoncé sera parfois considéré comme

${ }^{12}$ Pour une analyse détaillée de l'impératif en anglais, nous renvoyons le lecteur à Perrin (1992). 
subjonctif ou ontique. Nous commencerons donc par délimiter et légitimer la notion de «sujet» de l'énoncé de forme impérative, avant de décrire les différents types de groupes nominaux pouvant servir cette fonction.

\subsection{La distinction sujet / vocatif : schémas intonatifs}

Afin de différencier sujet et vocatif dans un énoncé de forme impérative, ce que nous ferons ici selon des critères uniquement intonatifs à l'exclusion des autres, il convient de tenter une caractérisation intonative des impératifs. Nos relevés personnels et les corpus transcrits présentés par O'Connor, Roach, et O'Connor et Arnold ${ }^{13}$ font ressortir un certain nombre de caractéristiques à l'étude de transcriptions telles que les suivantes:

(11) 'Sit down.

(12) 'Stop talking about it, Louis.

(13) 'Martha, 'you go a.way.

qui correspondent au schéma intonatif le plus couramment associé à un énoncé de forme impérative. On constate que dans tous les cas de figure,

- le head ${ }^{14}$, et le cas échéant le pre-head, se caractérisent comme high;

- le tail (vocatif ou groupe nominal remplissant une autre fonction, comme please, for Christ's sake, etc.) est systématiquement low;

- la dernière syllabe accentuée dans l'unité tonale constituée par le nucleus est marquée par une intonation descendante de type low fall, et s'il y a plusieurs syllabes accentuées, la première est high.

Cette caractérisation permet dans un premier temps de faire la distinction entre un énoncé de forme impérative et toute autre catégorie d'énoncés lorsque la structure morpho-syntaxique seule ne le permet pas, problème qui se pose uniquement dans deux cas : les «impératifs» nominaux (comme

\footnotetext{
13O'Connor, J.D. (1971): Advanced Phonetic Reader, Cambridge University Press, 52 p. Roach, P. (1983): English Phonetics and Phonology - A Practical Course, Cambridge University Press, 212 p. - O'Connor, J.D. et Arnold, G.F. (1973): Intonation of Colloquial English, second edition, Longman, 290 p. C'est dans ce dernier ouvrage que nous reprenons la terminologie et les notations techniques, [ . ] désignant une syllabe accentuée sans intonation propre, «nucleus» le noyau, «head» l'unité accentuelle précédant le nucleus et «pre-head» l'unité accentuelle précédant le head.

${ }^{14}$ Pour la définition de ces termes cf. note 13.
} 
Scalpel! ou The door!), dont nous ne traiterons pas ici étant donné la problématique choisie 15 ; et certains impératifs avec sujet $3^{\text {e }}$ personne que l'on pourrait confondre avec des énoncés de forme subjonctive ou déclarative au présent simple (i.e. quand l'absence de $-s$ à la $3^{\mathrm{e}}$ personne ne permet pas de décider s'il s'agit d'une assertion, contrairement à The tallest guy of you all step forward, où l'absence de $-s$ exclut forcément l'interprétation ontique).

En ce qui concerne la distinction subjonctif/impératif, il apparaît que la dernière syllabe accentuée du nucleus d'un énoncé au subjonctif ne se caractérise jamais par l'intonation descendante définitoire de l'impératif, mais soit par une intonation montante, soit par une intonation qui ne se distingue pas de celle de la précédente syllabe accentuée.

Dans le cas des énoncés de forme impérative pouvant être confondus avec un énoncé ontique, l'analyse est similaire. Ainsi, un schéma comme (14) impliquera un énoncé à l'indicatif, avec une nuance de reproche, alors que (15) sera forcément impératif :

(14) ^Some of you 'talk a lot. (and that bothers me)

(15) 'Some of you talk a lot. (so that John can hear us, OK?)

Dans les rares cas que nous avons relevés où subsiste une ambiguïté intonative, le contexte, tel que ceux fournis entre parenthèses, permet toujours de trancher.

Ayant ainsi circonscrit une classe d'énoncés proprement impératifs, voyons si une distinction indiscutable peut être faite au sein de cette classe entre les groupes nominaux employés comme vocatifs et comme sujets. Nous avons vu plus haut que le vocatif, qui se marquera généralement à l'oral par une pause et à l'écrit par une virgule, se distingue du reste de l'énoncé par son contour intonatif : il constitue une unité indépendante marquée par une intonation high.

Ceci nous permet de trancher entre deux interprétations de l'énoncé The tallest of you step forward selon les deux courbes intonatives suivantes:

(16) The 'tallest of you, 'step forward. (voc. + imp. sans sujet apparent)

(16')The 'tallest of you $\bullet$ step forward. (imp. ayant the tallest of you comme

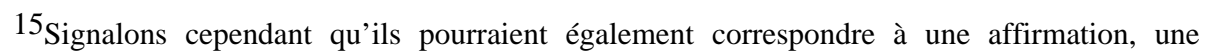
exclamation ou une interrogation selon le contexte, mais que chacune des quatre interprétations linguistiques envisageables se distingue des autres par un ton qui lui est propre.
} 
En effet, dans la mesure où step n'est pas porteur d'un ton particulier dans (16') mais simplement d'un accent tonique, il n'est pas la première syllabe accentuée du nucleus. Le nucleus inclut donc The tallest of you, qui ne peut être interprété que comme le sujet de step forward. En (16), en revanche, la situation est inverse : le high indiqué sur step indique le début du nucleus, ce qui implique que the tallest of you constitue une unité tonale indépendante, le head, et se verra donc interprété comme un vocatif.

Cette analyse étant applicable à tous les exemples des corpus que nous avons examinés, nous sommes à présent en mesure de travailler sur un ensemble d'énoncés que l'intonation permet de classer sans ambiguïté comme impératifs. Nous allons nous consacrer seulement à ceux comportant un groupe nominal sujet qui ne peut être confondu avec un vocatif.

\subsection{Les différents types de groupes nominaux sujet}

De nombreux linguistes soutiennent que les seuls groupes nominaux pouvant apparaître comme sujet d'un énoncé de forme impérative sont you et someone. Or la liste des autres types de groupes nominaux possibles est très longue. Nous en citerons ici quelques exemples seulement : pronom indéfini dans (17) à (19), groupe nominal déterminé par un quantifieur en (20) et (21), groupe nominal déterminé par the ou $\varnothing$ en (22) et (23), nom propre en (24)16 :

(17) Everyone move back.

(18) Don't anyone try to leave.

(19) Whoever saw the incident please come forward.

(20) One of you fetch some water.

(21) All competitors report to me.

(22) The man with the list come up here.

(23) Visitors please use the other entrance.

(24) John scatter the files and Bill ransack the desk.

Les sujets sont morphologiquement $3^{\text {e }}$ personne, certes, mais ils se caractérisent tous par le fait qu'ils réfèrent dans la situation co-locutoire à un sous-groupe des co-locuteurs, puisque, ainsi que le montre notamment (21),

16 $(17)$ et (18) sont tirés de Irving, J. (1972) The Water-Method Man, Black Swan, (1989, 262) (19) à (24) sont empruntés à Davies $(1986,133)$. 
on pourrait ajouter à chacun de ces groupes nominaux un partitif comme among you.

Par ailleurs, beaucoup d'énoncés de forme impérative, même si ce ne sont pas les plus fréquents, présentent un sujet qui englobe le co-locuteur et d'autres personnes (cf. (25) et (26)), ou même un sujet différent du colocuteur (cf. (27) et (28)) 17 :

(25) You and your men keep watch on the left while I get into position on the

(26) You and your friends get this mess cleared up before you go out.

(27) You go for help and the children stay here with me.

(28) You make the dinner and John do the washing up. No? All right then, John

On peut penser que ce qui rend ces exemples grammaticaux est la présence de deux impératifs conjoints où le premier a un sujet $2^{\mathrm{e}}$ personne, mais les deux exemples suivants ne rentrent pas dans cette catégorie :

(29) Those children of yours keep out of my garden or I'll set the dog on them.

(30) Your men guard the front while we creep round to the back.

Et si là encore on soupçonne que la présence du possessif your(s) soit la raison de l'acceptabilité, rappelons que des sujets $3^{\mathrm{e}}$ personne complètement détachés d'un $t u$ sont également possibles :

(31) Company C guard the front while Company D creep round the back, OK?

Le sujet potentiel peut donc être le ou les co-locuteurs, un sous- groupe des co-locuteurs, le co-locuteur et d'autres personnes, ou bien d'autres personnes. Remarquons que dans tous les exemples cités, même lorsque le sujet potentiel ne renvoie pas à un you, c'est bien au co- locuteur qu'est adressée la demande de validation éventuelle de la relation prédicative : l'impératif est une modalité dont le support est le locuteur, qui vise la validation d'une relation prédicative non encore validée, et dont il confie la prise en charge au co-locuteur (d'où la distinction possible entre le colocuteur, support potentiel de la validation de la relation prédicative, et le sujet, actant potentiel du procès).

Dans (27), par exemple, You go for help and the children stay here, nous avons deux impératifs. Pour les deux, c'est au co-locuteur que revient la validation éventuelle de la relation prédicative. Mais si le sujet du premier est right.

cook and you wash up.

(dans un contexte où l'on ne s'adresse $\mathrm{p}$

${ }^{17}$ Repris à Davies (1986, 140-141). 
you, celui du deuxième est uniquement the children. De même en (31), la prise en charge du lien S (Company C) / P ( guard the front) est offerte au colocuteur (you), qui devra se charger de donner l'ordre «direct» aux deux compagnies en question.

Le problème central est alors de voir pourquoi, si nous acceptons l'hypothèse selon laquelle l'impératif est une modalité déontique excluant certaines opérations de construction référentielle, l'anglais accepte la présence d'un sujet $3^{\mathrm{e}}$ personne référentiellement dissociable du co-locuteur, seul paramètre permettant pourtant une construction référentielle en prise directe avec la situation de co-locution sans intervention d'un accord linguistique co-énonciatif.

\section{Analyse}

Pour déterminer la fonction exacte du sujet de l'énoncé de forme impérative en anglais, et voir si elle est compatible avec le fonctionnement théorique proposé pour le français, nous partirons du cas qui se rapproche le plus du français, l'impératif sans sujet apparent, car il nous fournira une base «dépouillée» pour analyser a contrario mais avec les mêmes outils théoriques les cas où l'énoncé de forme impérative comporte un sujet apparent.

\subsection{L'impératif sans sujet apparent : une forme neutre}

Si aucun sujet n'apparaît dans un énoncé de forme impérative, l'actant potentiel est forcément identifiable au co-locuteur, puisque, sauf mention contraire, tous les calculs de repérage d'un énoncé impératif se font directement par rapport à la situation de co-locution. Pour preuve de cette affirmation, rappelons que dans un impératif sans sujet apparent, un pronom personnel, possessif ou réfléchi introduit à des fins d'anaphore coréférentielle au sujet sous-entendu ne peut être que $2^{\mathrm{e}}$ personne :

(32) Speak his name and you sentence him to death.

(33) Calm down, will you?

(34) Put a tiger in your tank. 
(35) Judge every one and everything for yourself. 18

Nous rejoignons ici Dobrovie-Sorin (1981), selon qui «la forme impérative ne se construit pas par rapport à un $t u$ «profond» qui serait effacé en surface. La position sujet est vide, non instanciée, et repérée directement par rapport» au co-locuteur, à cette différence près que, selon nous, c'est précisément parce que le sujet est repéré par identification au co- locuteur que la place sujet est vide. En effet, si cette absence de sujet désigne implicitement l'allocutaire comme sujet potentiel, c'est parce que la nature déontique de l'impératif interdit tout repérage autre que co- locutoire. Mais si la place de sujet reste vide c'est bien parce que, dans la mesure où l'éventuelle validation de la relation prédicative échappe au locuteur, il ne peut y avoir «forçage de consensualité énonciative» permettant l'identification visible du co-locuteur à l'argument sujet. Tout ce que dit un impératif sans sujet apparent, c'est que l'actant potentiel du procès que je vise est l'autre de la dyade co-locutoire (cf. les you de reprise dans les énoncés (32) à (35)); mais la nature déontique de l'impératif nous empêche d'assigner une référence linguistique construite à cet argument sujet dont on ne peut guère que donner la valeur en creux.

Cette analyse correspond strictement au fonctionnement du français, à cette différence près que, bien naturellement, l'anglais n'a pas recours à une marque de personne pour signifier la différence singulier/pluriel19. Reste alors à voir si, dans la mesure où le fonctionnement opératoire de base de l'impératif semble identique en français et en anglais, la possibilité d'apparition d'un sujet remet en cause cette convergence fondamentale.

\subsection{Le degré zéro de la construction référentielle}

Si l'on admet que lorsque le sujet n'est pas spécifié dans l'énoncé de forme impérative, on se trouve dans un cas neutre où il est identifiable au colocuteur, alors on n'utilisera un sujet apparent que pour marquer en surface une opération supplémentaire de construction référentielle. Or toute

\footnotetext{
18(32) in The Three Musketeers de George Sidney (1948). (33) in Twelve Angry Men de Sidney Lumet (1957). (35) in James, H. (1881): The Portrait of a Lady, Penguin Books (1984, 300).

${ }^{19}$ Nous excluons volontairement de cette étude le cas des énoncés de forme impérative comportant let, qui fera l'objet d'un article distinct. Signalons cependant que les résultats de nos analyses ne sont pas de nature à remettre en cause les conclusions ici présentées.
} 
construction référentielle impliquant un accord co-énonciatif est impossible à l'impératif, puisqu'on est dans le cadre d'une modalité déontique.

Le premier élément nous permettant de réduire ce paradoxe nous est apparu dans une remarque faite par Dobrovie-Sorin $(1985,53)$ :

«Dans les langues modernes, la $3^{\mathrm{e}}$ personne se trouve hors du domaine énonciatif. Tel n'était pas le cas du latin, qui présentait deux formes de pronoms pour la $3^{\mathrm{e}}$ personne : une forme "anaphorique" et une autre "personnelle", liée au domaine de l'énonciation. L'existence de l'impératif de la $3^{\mathrm{e}}$ personne en latin pourrait être reliée à l'existence de cette forme personnelle de la $3^{\mathrm{e}}$ personne du pronom.

En effet, si l'on conçoit aisément qu'une référence nominale construite par anaphore soit incompatible avec l'impératif, dans la mesure où toute opération seconde implique une certaine consensualité exclue par la déonticité de cette modalité, l'on peut en revanche imaginer des constructions référentielles par désignation renvoyant directement à la situation de colocution. En d'autres termes, autant un groupe nominal $3^{\mathrm{e}}$ personne dont la référence est construite au prix d'un certain accord co-énonciatif ne sera jamais acceptable comme sujet d'un impératif, autant l'impératif est compatible avec une désignation référentielle qui, quoique grammaticalement $3^{\text {e }}$ personne, n'est pas extérieure à la situation de co-locution et ne nécessite pas d'accord entre le locuteur et le co-locuteur sur le monde qui les entoure pour être jugée linguistiquement existante.

L'impossibilité d'apparition de certains sujets $3^{\mathrm{e}}$ personne dans un énoncé de forme impérative tiendrait donc uniquement au fait que leur référence n'est pas construite par repérage direct dans la situation de co- locution, mais par une construction référentielle au sens strict du terme, ce qui implique un accord co-énonciatif. C'est notamment le cas des pronoms personnels $3^{\mathrm{e}}$ personne (* He / They open the door.) ou des groupes nominaux $3^{\mathrm{e}}$ personne construits par anaphore contextuelle (* The guy you mentioned stop calling you, $O K$ ?). Mais il suffit, comme pour le pronom $3^{\mathrm{e}}$ personne non anaphorique du latin, que la référence du groupe nominal en question puisse être automatiquement fournie par la situation de co-locution pour que le problème soit éliminé.

Or tous les groupes nominaux $3^{\mathrm{e}}$ personne pouvant être sujet d'un énoncé de forme impérative en anglais ressortissent de ce fonctionnement. Ceci est 
évident pour ceux qui renvoient à un sous-groupe des co-locuteurs (One of you, some of you, whoever, all competitors, the man with the list, the tallest of you, visitors, etc.). Le sujet sert alors à marquer la valeur de l'argument sans en construire la référence exacte, et les déterminants, quand déterminants il y a, sont légitimés par une justification exclusivement situationnelle. Il n'y a pas ici recours à la sphère co-énonciative, et le sujet se borne à exprimer une valeur de localisation par rapport à la situation de colocution (cf. l'introduction possible à chaque fois de among you, of you ou there), c'est-à-dire le décalage par rapport à une situation neutre où il y a identification référentielle stricte entre sujet et co-locuteur(s).

C'est également le cas pour les impératifs dont le sujet est un nom propre. Nous avons cité l'énoncé John scatter the files and Bill ransack the desk, correct dans la mesure où John et Bill représentent deux sous-parties de l'ensemble des co-locuteurs constitué par [ John + Bill ], et non des noms propres construits comme $3^{\mathrm{e}}$ personne dans une co- énonciation, ce qui serait le cas de *John scatter the files si l'on parle à Bill (John étant absent) 20 .

Restent enfin les quelques cas que nous avons signalés où le groupe nominal $3^{\mathrm{e}}$ personne n'est repéré ni par identification ni par localisation par rapport au co-locuteur, comme Company $C$ guard the front while Company $D$ creep round the back, par exemple. Nous postulons ici, l'ayant démontré précédemment, que ce type d'énoncé n'est acceptable que lorsque la situation de co-locution implique la possibilité d'existence d'une autre situation de colocution dans laquelle le co-locuteur de la première deviendrait locuteur, avec pour co-locuteur le sujet de l'énoncé primaire. Ainsi, l'énoncé de forme impérative analysé n'est possible que dans la mesure où l'officier auquel s'adresse le locuteur est susceptible de donner l'ordre «direct» aux deux compagnies en question. Là encore, c'est la possibilité de rester dans une situation de co-locution où les calculs de repérage peuvent se faire nonanaphoriquement, sans passage obligé par un accord co-énonciatif sur les références, qui explique la grammaticalité.

A ce stade de notre étude, il apparaît que l'hypothèse avancée sur le fonctionnement de l'impératif comme modalité discursive essentiellement

${ }^{20}$ De même, John, scatter the files si l'on ne parle qu'à John ne serait acceptable que dans la mesure où John est un vocatif placé avant un impératif sans sujet apparent (cf. supra). 
déontique et donc co-locutoire s'applique aussi bien au français qu'à l'anglais, et cela que l'énoncé anglais comporte une place vide de sujet ou un sujet $3^{\text {e }}$ personne.

\subsection{Retour du co-locutoire au co-énonciatif}

Le seul cas jusqu'à présent négligé est celui des énoncés de forme impérative où le sujet, pourtant $2^{\mathrm{e}}$ personne, est explicitement exprimé par un you. Contrairement à ce que laissait entendre notre analyse de 5.1., selon laquelle ce cas de figure correspond à une situation neutre où le sujet n'est pas exprimé, ces énoncés sont parfaitement possibles, et même très courants.

En fait, il semble qu'il y a alors préconstruction par le locuteur d'un désaccord co-locutoire sur la valeur de l'argument sujet, due le plus souvent à la prise en compte d'un contexte antérieur. Cette insistance contrastive est particulièrement évidente dans les dialogues suivants 21 :

(36) «Don't be ridiculous,» Homer said. «She just knows - she always knows.» «Don't you be ridiculous,» Candy said crossly.

(37) «You just have to scribble a few lines on this card.» "You scribble something, Maggie.»

(38) Emerald, thin-lipped : «But be a little respectable... the family name...» At which Pia tossed her head. "You be respectable, sister,» she said.

Quand le locuteur réintroduit ainsi le you, il fait porter l'impératif sur une relation prédicative déjà soudée, pour ainsi dire, ce qui supprime l'autonomie possible du co-locuteur 22 . Et de fait, il y a «forçage de consensualité coénonciative», comme dans le cas de «Tu manges ta soupe!» en français.

21(36) in Irving, J. (1985): The Cider-House Rules, Black Swan, (1989, 609); (37) in Williams, T. (1956): Cat on a Hot Tin Roof, Penguin Books, (1976, 28); et (38) in Rushdie, S. (1981): Midnight's Children, Picador, (1982, 329).

${ }^{22}$ Signalons au passage que le fonctionnement est exactement identique lorsque, face à un ensemble de co-locuteurs, l'énonciateur utilise all of you ou everyone, comme dans All of you listen to me!, ou Everyone stay where they are. Le locuteur préconstruit la non-validation de la relation prédicative en raison d'une hétérogénéité de la classe. Dire All of you listen to me, c'est présupposer que tous les co-locuteurs ne sont pas à l'écoute, et marquer le sujet est alors un moyen de recréer l'homogénéité de la classe en prenant en compte cette présupposition directement dans l'énoncé, par préconstruction. 
Voici donc un cas où la dissociabilité des notions de co-locuteur et coénonciateur telles que nous les avons présentées en A. joue à plein, puisque si, dans le cas d'un impératif $2^{\mathrm{e}}$ personne sans sujet apparent, la valeur de l'argument sujet se construit par rapport à la situation de co-locution, le you n'apparaît dans l'énoncé impératif que lorsque le locuteur veut marquer un désaccord avec celui qu'il faut bien alors appeler un co-énonciateur. La construction de référence ne peut se faire en fonction de la seule situation de co-locution : elle passe par une remise en question d'un (dés)accord coénonciatif thématique.

Il ne nous semble pas cependant que ce cas limite, qui fait intervenir pour une certaine part les fonctionnements co-énonciatifs dans le jeu co-locutoire propre à l'impératif, remette en question les hypothèses ici discutées sur la déonticité de l'impératif. En effet, de même que l'on peut avoir recours à un vocatif dans un plan de discours essentiellement déclaratif (cf. §3.1.2.), de même l'on peut, pour des motifs argumentatifs précis, glisser du co-locutoire au co-énonciatif lorsque le contexte avant impose une certaine thématicité de l'argument sujet. Car, rappelons-le, ce you thématique intervient bien pour marquer un désaccord co-énonciatif, c'est-à-dire certes une opération sur un accord entre co-énonciateurs, mais une opération qui est précisément la destruction de cet accord préalable.

\section{Conclusion}

En dernière analyse, donc, il apparaît que l'hypothèse avancée pour l'impératif en français vaut également pour l'anglais, et cela que les énoncés de forme impérative considérés comportent ou non un sujet, celui-ci pouvant indifféremment être $2^{\mathrm{e}}$ ou $3^{\mathrm{e}}$ personne dans les limites des critères ici définis. L'explication des différences syntaxiques relevées entre les deux langues (possibilité d'apparition d'un sujet en anglais, fonctions respectives du vocatif en français et du sujet en anglais,...) semble résider dans la différence morpho-syntaxique de base entre ces deux langues : en français, l'impératif est un «mode» marqué par des désinences propres qui activent déjà un sujet en puissance (tu, nous ou vous), impliquant l'un ou les deux pôles de la dyade co-locutoire. En anglais, en revanche, l'utilisation de la base verbale nous place en deçà de cette présélection, et laisse le champ relativement libre pour l'utilisation d'un sujet apparent, dans la mesure où la construction 
référentielle de celui-ci ne contrevient pas à la déonticité fondamentale de l'impératif.

\section{Bibliographie}

Brunot, Ferdinand et Bruneau Charles (1969 rééd.): Précis de grammaire historique de la langue française. Paris: Masson, 322-323.

Curvat, Hervé (1991): Morphologie verbale et référence temporelle en français moderne. Essai de sémantique grammaticale. Genève-Paris: Droz, 151-170.

Damourette, Jacques/Edouard Pichon (1911-1940): Des mots à la pensée. Essai de Grammaire de la Langue Française. Paris: D'Artrey, 1968 (rééd.). Tome I §48-57, 73, 438 et 442 , Tome II $\S 741,765-766$ et 781 , Tome III $\S 1021$, Tome IV $\S 1382-1411$ et 1431-1448, Tome VI § 2175-2178.

Davies, Eirlys (1986): The English Imperative, Croom Helm.

Dobrovie-Sorin, Carmen. (1981): A propos de l'interprétation des groupes nominaux indéfinis dans les structures impératives. In: Semantikos, vol. 5, n_1, 55-69.

Dobrovie-Sorin, Carmen (1985): Actes de langage et théorie de l'énonciation, DRL, Université de Paris VII, coll. ERA 642.

Nyrop, Kristian (1930): Grammaire historique de la langue française. Copenhague: Gyldendalskee Boghandel Nordisk Forlag, 1960 (rééd.). Tome deuxième, 119-122 et Tome sixième, 270-275.

Perrin, Isabelle (1992): L'Impératif en anglais et les opérations énonciatives, doctorat nouveau régime, Université de Paris III.

Wagner René-Léon (1974): L'ancien français. Paris: Larousse, 183-201. 